\title{
MANIFESTATIONS POSSIBLES DE LA COURONNE DE CHAPMAN DANS LA RADIOASTRONOMIE
}

\author{
F. LINR \\ Institut astronomique de l'Académie des Sciences \\ Prague, Tchécoslovaquie
}

On peut se demander si la grande extension de la couronne solaire demandée par l'hypothèse de Chapman [1] pourrait se manifester par les phénomènes de réfraction lors d'une occultation, par exemple celle de la source du Taureau.

Une thérie simplifiée de la réfraction électronique peut donner une estimation de ce genre. Nous admettrons que la concentration électronique de la couronne $N$ varie dans un certain intervalle des distances suivant la formule $N=a \rho^{-n}$, où $\rho$ est la distance au centre du soleil exprimée en rayons de celui-ci et $n$ un exposant positif. Dans ces conditions la déviation totale $\omega$ du rayon passant à la distance minimum $\rho_{0}$ sera donnée par [2] $\omega=B a \rho_{0}^{-n} f^{-2}=B N_{0} f^{-2}$, où $f$ est la fréquence exprimée en $\mathrm{Mc} / \mathrm{s}$ et $B$ un facteur qui est fonction de $n$ et que l'on trouve dans le tableau suivant:

$\begin{array}{lccccccc}n & 0 & 1 & 2 & 3 & 4 & 5 & 6 \\ B & 0.00 & 0.28 & 0.43 & 0.55 & 0.65 & 0.74 & 0.81\end{array}$

Dans le cas le plus favorable la structure de la couronne de Chapman $\left(T_{0}=1.2 \times 10^{\circ}\right)$ vers $\rho_{0}=18$ sera donnée par $N=1.5 \times 10^{5} \rho(n=1)$ et la réfraction y sera pour les différentes fréquences:

$\begin{array}{lccccc}f & 10 & 17.5 & 24.5 & 31.6 \mathrm{Mc} / \mathrm{s} \\ \omega & 30 & 10 & 5 & 1 \text { minutes d'arc } \\ 8^{-1} & 1.01 & 1.001 & 1.000 & 1.000\end{array}$

En dernière ligne est encore donnée la modification de l'intensité par la refraction $s^{-1}=I / I_{0}$ calculée par

$$
s=1-(n-1) \frac{\omega}{R_{\odot} \rho}-n\left(\frac{\omega}{R_{\odot} \rho}\right)^{2} .
$$

Il est alors douteux que la déviation ainsi que l'augmentation de l'intensité puissent être décelées par nos moyens actuels d'observation.

\section{REFERENČES}

[1] Chapman, S. Smithson. Contr. Astro. 2, No. 1, 1957.

[2] Link, F. Bull. Astr. Inst8. Csl. 3, 6, 1952. 